\title{
MODELLING AND CONTROL OF A BIPED ROBOT $^{\star}$
}

\author{
N.F. Gonçalves ${ }^{1}$ P.M. Silva ${ }^{1}$ J. Sequeira ${ }^{2}$ \\ M.I. Ribeiro ${ }^{3}$
}

\author{
Instituto Superior Técnico/Institute for Systems and \\ Robotics, Av Rovisco Pais 1, 1049-001 Lisboa, Portugal
}

\begin{abstract}
:
This paper presents a study on two kinematic structures for dynamically stable biped locomotion. The two legs, each with three degrees of freedom, are connected through a rigid waist. The dynamics of both structures is modelled by partitioning the six dof kinematic chain in a 3 dof supporting leg and a 3 dof balance leg. A low complexity model is obtained by simplifying the dynamics of the balance leg not accounting for rotational dynamics. It is shown that the overall model produces good results under the point mass assumption for each link and a standard robust control algorithm.
\end{abstract}

Keywords: Humanoids, Legged robots, Biologically inspired robots

\section{INTRODUCTION}

Biped robots are currently a hot research topic in robotics. State of the art bipeds are close to the point where they can be used to replace humans in a variety of tasks taking place in environments designed for humans (as the typical industrial environment). Legged locomotion is characterized by the existence of multiple sets of ground contact points that generate identical postures. This characteristic is specially useful in irregular terrains as the robot posture is less constrained by the obstacles (if one set of ground contact points is not feasible due to an obstacle then it is, in general, possible to use alternative sets) as opposed to wheeled locomotion with its fixed contact points that can not avoid obstacles. Biped robots combine the flexibility of legged locomotion with a small number of ground contact points (simplifying the design of locomotion strategies) at the expense of added difficulties in motion stability. Furthermore,

\footnotetext{
* This work was partially supported by the FCT Programa Operacional Sociedade de Informação (POSI) in the frame of QCA III.

1 nelsongoncalves@zmail.pt

2 jseq@isr.ist.utl.pt, corresponding author

3 mir@isr.ist.utl.pt
}

biped robots are socially relevant as they can be used to develop new artificial limbs for the handicapped.

Biped robots are usually studied aiming at emulating human like locomotion capabilities, (Gill A. Pratt, 2000; Youji Nakajima et al., 2000; Atsuo Takanishi et al., 1990), although animal biped locomotion deserved also some attention, (J. Pratt and G. Pratt, 1998). Human locomotion is characterized by two distinct phases: the swing phase and the double support phase. In the swing phase, one foot, the supporting foot, is fixed on the ground, whereas the other foot, the balance foot, swings forward fast enough to avoid tip over. When the balance foot hits the ground, starts the double support phase. In this phase both feet are on the ground and exchange roles, with the supporting foot becoming the balance foot for the new step. The double support phase ends when the balance foot leaves the ground.

One of the main issues in biped locomotion is stability. Static stability requires that the biped be stable in any static configuration and its measure depends exclusively on position data. The complementary concept is dynamic stability which requires that the biped does not fall down while walking, without being concerned with 
the stability at each point of the trajectory. Dynamic stability measures depend also on velocity data. To circumvent dynamic stability issues, some biped structures have counterweight devices. In humans, this counterweight role is performed by the upper body, including arms and trunk. The structures considered in this paper have a small number of degrees of freedom (dof) when compared with the kinematics of a human body. Small dof biped structures are simple to model and build, though requiring, in general, more sophisticated control techniques. Furthermore, the reference motion needs to be carefully chosen to achieve dynamic stability.

Two kinematic structures are considered in this paper, both composed of a pair of legs connected through a rigid waist. No counterweight devices are used. The bipeds move only on the sagital plane and in an obstacle free environment. The point mass assumption greatly simplify the dynamic models. Nevertheless, it remains as a valid hypothesis for realistic modelling if the masses and physical dimensions of the links involved are carefully designed. Furthermore, for the considered structures, the point mass assumption avoids lateral stability problems and hence the overall stability can be controlled by a trial-and-error procedure on the step size.

The dynamic models of both structures were obtained using the Newton-Euler iterative algorithm, (J. Craig, 1989), with the coupling between legs being aproximated by the motion of a rigid body. Structures similar to the ones considered in this paper are presented in (Ching-Long Shi, 1999), using prismatic joints and a counterweight mass, and in (Eturoo Igarishi and Tooru Nogai, 1992), using revolution joints without a waist. Alternative kinematic structures, using counterweight devices to improve stability, are reported in (Ching-Long Shi, 1999; Atsuo Takanishi et al., 1990; C. Chanchai and C. Prabhas, 2001). Human body replica structures are reported in (Gill A. Pratt, 2000; Youji Nakajima et al., 2000; J. Pratt and G. Pratt, 1999).

The remainder of this paper is organized as follows. In Section 2 the two kinematic structures considered are described. The corresponding dynamic models are presented in Section 3. In Section 4 simulation results are presented for motion along the sagital plane in obstacle free environment. Finally, Section 5 concludes the paper and presents directions for further research.

\section{KINEMATIC STRUCTURES}

The two different kinematic structures considered have two legs and a rigid connecting waist. Given that the biped locomotion considered in this paper is constrained to the sagital plane, the legs need only to move in this plane and hence each of them has a planar structure.

The kinematics of the first structure is illustrated in Figure 1. All the joints are of revolution type. Each leg has three joints (all of them actuated), identified with the human leg: hip joint, knee joint and ankle joint.
In the second structure, the knee joint is replaced by a prismatic joint. The corresponding diagram is shown in Figure 2.

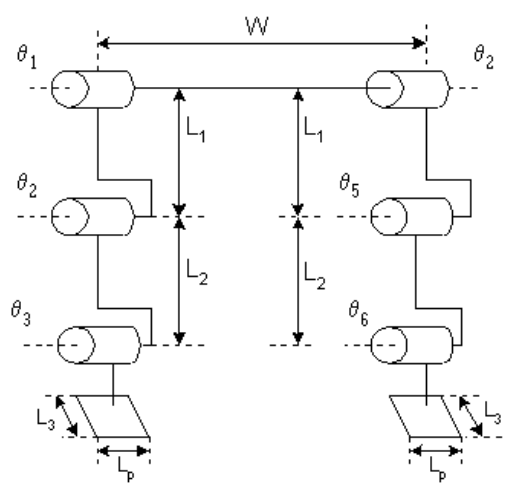

Fig. 1. Structure 6R

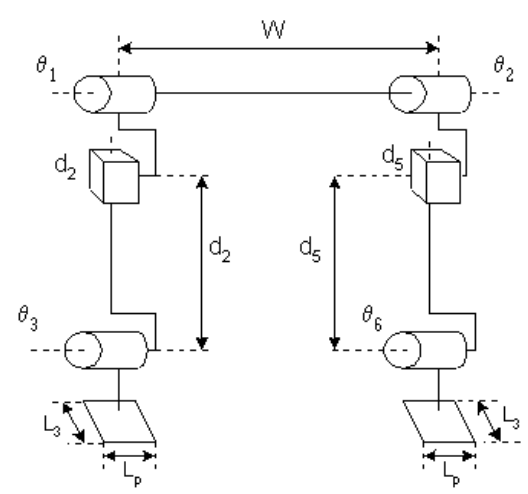

Fig. 2. Structure RPRRPR

In the $6 \mathrm{R}$ structure the three joints are used to position and orientate the foot, whereas in the RPRRPR structure the knee joint is used only in positioning the foot with the ankle joint used mainly for the foot orientation. For this reason, specifying a reference motion for the structure RPRRPR is simpler than for structure $6 \mathrm{R}$.

With a single foot on the ground, both structures are statically unstable, meaning that they loose balance and tip over if kept long enough in an unstable configuration. To overcome this difficulty, additional dof allowing the motion of the legs, and hence of the center of mass of the robot in the transversal plane, can be used, (Youji Nakajima et al., 2000). To avoid high torques in the hip joint, balance masses moving in the sagital plane are also used to move the center of mass of the robot, (ChingLong Shi, 1999; Youji Nakajima et al., 2000; C. Chanchai and C. Prabhas, 2001). Balancing strategies may require elaborate mechanical structures to accomodate the corresponding sensors and actuators. The dynamics model reflects this added mechanical complexity by increasing the importance of nonlinear terms. Furthermore, balance masses tend to be important when compared with the rest of the structure, and hence actuators in the raw locomotion structure are required to generate increased 
power, e.g., the upper body in humans, where the balance dof lie, represents an average of $50 \%$ of the total mass.

\section{DYNAMICS}

Legged locomotion is characterized by the leg-ground interactions occuring at sparse instants of time. In general, for statically stable robots, the interactions occuring at leg taking off and landing can be safelly ignored in a dynamics model, this not being the case when large energy transfers are involved. However, for statically unstable structures, such as the ones in this paper, even low power leg-ground interactions propagate throughout the whole structure and therefore must be taken into account when designing the control system as they may not yield a robust walking, (Yasutaka Fujimoto and Atsuo Kawamura, 1998). Assuming that no slippage occurs between the feet and the ground, the relevant leg-ground interaction takes place during the double supporting phase at every impact on the ground.

\subsection{Modelling of the two structures}

The modelling of each of the structures takes advantage from the fact that both are serial kinematic chains and hence standard procedures on the kinematics and dynamics modelling of serial manipulators can be used (6-dof serial manipulators for each of the structures considered in the paper). This procedure identifies the base of the manipulator with the support foot and the end-effector of the manipulator with the balance foot. The impact and ground reaction forces are modelled as external forces acting on the tip of the manipulator, i.e., the balance foot.

To obtain the dynamics model for robotic manipulators, the Newton-Euler or the Lagrange methods are commonly used. For manipulators with more than three dof, these methods lead to expressions for the dynamic model that may be hard to analyse, (Carlos Canudas de Wit et al., 1997).

To overcome the difficulties in obtaining the dynamics model for the 6-dof serial structures, a partitioning strategy schematically represented in Figure 3 is proposed.

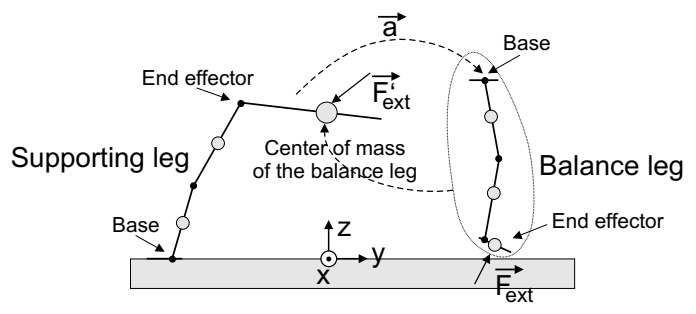

Fig. 3. Biped modelling through partitioning

This strategy considers each of the structures as being composed by three separate components. Both legs can be modelled as serial 3-dof manipulators. For the supporting leg, the base and tip of the serial manipulator are identified, respectively, with the supporting foot and the waist. For the balance leg, the base is identified with the waist whereas the tip is identified with the balance foot. The influence of the supporting leg on the balance leg is accounted by setting: (i) the linear and angular acceleration of the base of the balance leg equal to the linear and angular acceleration of the tip of the supporting leg (the waist is assumed to be a rigid body), (ii) the mass of the tip as the sum of the masses of the waist and balance leg, and (iii) the center of mass of the tip as the composition of the centers of mass of the waist and of the balancing leg.

At each time instant, the supporting leg sees the balance leg as a rigid body, not accounting for the corresponding rotational dynamics. The forces/torques acting on the tip of the supporting leg are computed straight from those acting on the balance foot. The dynamics of the balance leg tends to be relevant for some combinations of the masses (distribution and value) and of the length of the links. For instance, a long balance leg tends to generate a large torque at the waist, thus requiring the adequate reaction from the controllers in the joints of the supporting leg to avoid excessive rolling.

Regardless of this oversimplification, this strategy has the non negligible advantage of reducing the complexity of the dynamics model. As already referred, the use either the Newton-Euler (the technique used in this paper) or the Lagrange formulations to express the exact dynamics of a 6 -dof kinematics chain leads to complex expressions. Under the point mass assumption, the lateral stability of both structures is easily verified as the involved torques have no sagital component. The overall stability can thus be reduced to the sagital stability which in turn can be controlled by setting the step size to a small enough value.

\subsection{Modelling impact and ground reaction forces}

In the double supporting phase, both legs support the robot. Depending on the motion of the joints while in this phase, the ground reaction felt by each foot changes. Note that in a serial manipulator not subject to any external forces the ground reaction occurs only at the base and is constant, assuming no changes in the weight of the manipulator. Figure 4 shows how to compute the ground reaction force, $\overrightarrow{R_{2}}$, acting on the balance foot.

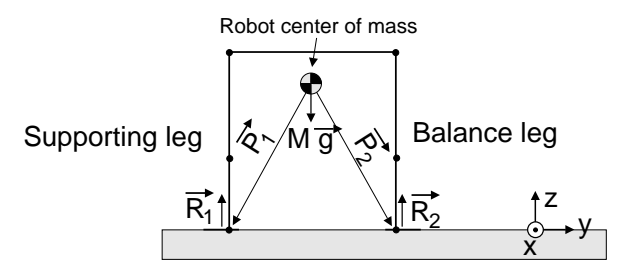

Fig. 4. Ground reaction modelling (identical for both structures) 
In the double supporting phase, the only forces acting on the robot center of mass are the gravity and the ground reaction forces. Assuming that the robot is in equilibrium, the resulting torque acting on the robot is zero. These conditions are expressed by (1), where $M$ is the robot total mass, $\vec{g}$ is the gravity acceleration and $\overrightarrow{R_{1}}$ and $\overrightarrow{R_{2}}$ are the ground reaction forces represented in Figure 4.

$$
\overrightarrow{R_{1}}+\overrightarrow{R_{2}}=M \vec{g}, \quad \overrightarrow{P_{2}} \times \overrightarrow{R_{2}}+\overrightarrow{P_{1}} \times \overrightarrow{R_{1}}=0
$$

The balance phase ends with the impact of the balance foot on the ground. During this phase the robot is falling as it is statically unstable (in both structures). If not properly controlled it may not be able to gently place the balance foot on the ground (and thus not minimizing the perturbations that will be induced in the whole structure). In general, the ground has some complacency meaning that, on impact, the balance foot does not stop immediately. The impact forces are modelled by elastic systems, arranged as in Figure 5 where $K_{1}$ and $K_{2}$ are the elastic spring coeficients and $\vec{v}_{\text {impact }}$ is the impact velocity vector. The severity of the impact can be modified acting on the spring constants. This model is exclusively used to compute the impact forces and not the ground reaction forces.

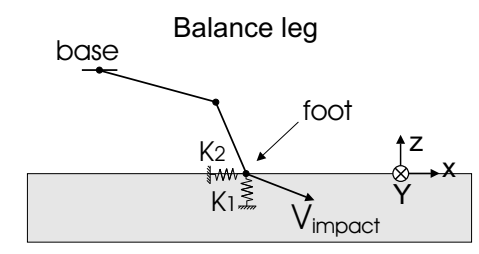

Fig. 5. Impact forces model

The impact forces acting on the balance foot are described by

$$
\begin{aligned}
\vec{F}_{\text {impact }}=\left(\Delta_{x} K_{2}, 0, \Delta_{z} K_{1}\right) & \\
& \text { if } \Delta_{x} \neq 0 \vee \Delta_{z} \neq 0
\end{aligned}
$$

where $\Delta_{x}$ and $\Delta_{z}$ are the displacements of the foot on impact. The total generalized forces acting on the balance foot are thus

$$
\vec{F}_{\text {ext }}= \begin{cases}\vec{F}_{\text {impact }}+\vec{R}_{2} & \text { if foot on the ground } \\ 0 & \text { otherwise }\end{cases}
$$

\section{EXPERIMENTAL SIMULATION RESULTS}

The dynamics model obtained from the application of the standard iterative Newton-Euler algorithm, together with the simplifying assumptions and the models for the ground reaction and impact forces described in Section 3, was simulated in Matlab. The reference trajectories for each of the joints in the robot were synthesized from the kinematics model motion that generates human like locomotion.

Table 1 shows the physical parameters data for both robots.

\begin{tabular}{ccc}
\multicolumn{3}{c}{ Dimensions $(\mathrm{cm})$} \\
\hline$L_{1}=15.5$ & & $L_{p}=4$ \\
$L_{2}=15.5$ & & $W=25$ \\
$L_{3}=10$ & & $d_{0}=30$ \\
\hline & & \\
Body & Links & Mass $(\mathrm{Kg})$ \\
\hline Legs & waist-knee & 0.5 \\
& knee-foot & 0.5 \\
& foot & 0.5 \\
Waist & & 0.5 \\
\hline
\end{tabular}

Table 1. Robot dimensions and weight.

For the presented experiments, the step size is $0.1 \mathrm{~m}$ and the planned duration is $0.1 \mathrm{~s}$. This relatively fast motion $(1 \mathrm{~m} / \mathrm{s})$ is imposed by natural static instability of the simple kinematics of both structures, requiring the balance foot to reach the floor as quickly as possible to prevent the robot from falling down.

For each leg, a 3-state automaton chooses the control mode according to the actual phase of the system: supporting, if the leg is in the supporting phase, balance, if the leg is in balance phase or double supporting if both feet are on the ground. The duration of the double supporting phase is determined by the need to stabilize the robot when both feet are on the ground. Stabilizing periods are required at the begining and end of the motion, and in the interval between the end of a step and the begining of a new one (note that, a standing up $6 \mathrm{R}$ structure biped robot resembles a double inverted pendulum like dynamics). At each of the states, a continuous time standard partitioned robust controller, (Carlos Canudas de Wit et al., 1997), is used to generate the control for the actuators in the joints.

Figure 6 shows the motion of the robot over time using a stick diagram (each link is represented by a line) for a set of 4 steps. The diagram shows the biped motion with the dynamics model described in the previous sections.

In the initial stage of the step, after an initialization procedure, the robot lifts the balance leg, bending the knee, while the supporting leg starts pushing the waist forward. In the second stage, the supporting leg continues to push forward while the balance leg further extends the hip link and unwinds the knee forward. In the last stage, the robot places the balance leg on the ground, recovers the original height of the waist and stops for a period of time long enough to recover from the impact of the balance foot on the ground and to achieve a stable posture (an empirical $25 \%$ of the step duration was used in the experiments). After this, a new step begins with the roles of the support and balance legs interchanged.

For the revolution structure, Figure 7 shows the torques applied by the joint actuators at the support and balance legs. The robot is switched on at $t=0$ while in double support mode, with both legs in the upright position (the initial step position). An initial 0.05s time interval is used to achieve a stable posture. The torques of the hip joints in both legs show a transient behavior lasting for about 0.01s. For the balance leg, the knee joint also 


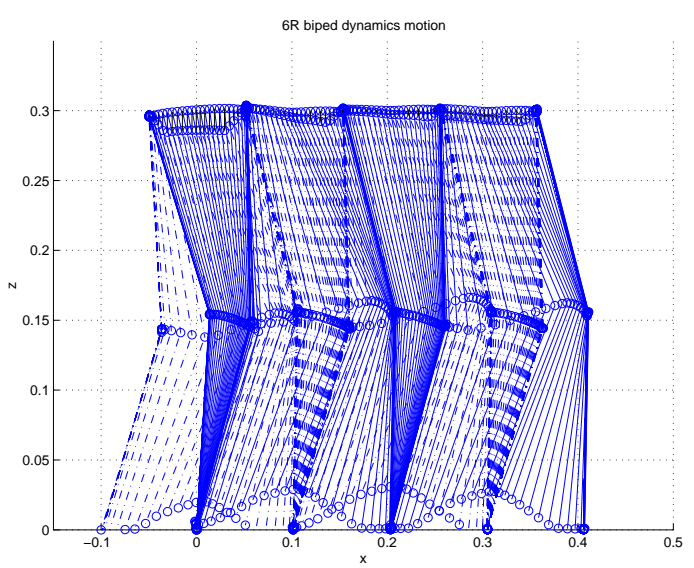

Fig. 6. Stick diagram for the $6 \mathrm{R}$ structure locomotion

exhibits a transient behavior whereas the supporting leg knee is almost not affected.

The step motion starts at $0.05 \mathrm{~s}$ and the torques in Figure 7 show, roughly, four relevant time slots, corresponding to the intervals 0.05 to $0.075 \mathrm{~s}, 0.075$ to $0.1 \mathrm{~s}, 0.1$ to $0.13 \mathrm{~s}$ and 0.13 to $0.15 \mathrm{~s}$.

During the first interval, the supporting leg rotates slightly to push the robot forward, this motion being made, mainly, by the ankle and hip joints. The balance leg starts raising and hence the hip and ankle joints in the supporting leg must counteract the tendency for the robot to tip over, this justifying the negative torques. During this period the knee motion is limited to a slight bend. Given this limited motion, the ankle joint "sees" the remaining of the robot almost like the hip joint and hence the similarity of their respective torques. The raise of the hip by the balance leg is followed by the bending of the balance knee, so that there is enough space for the robot to account for the forward motion due to gravity. While in the air, the balance foot is not influenced by any external forces/torques and therefore the dynamics of the hip joint is similar to that of the knee joint leading to similar patterns for the joint torques (the difference in the sign being due to the opposite rotations performed by each of them).

During the interval 0.075 to $0.1 \mathrm{~s}$, the supporting leg effectively pushes forward the whole structure, whereas the balance leg moves forward, extending its knee (previously bended) and preparing for the impact with the ground. During this period, the hip joint in the balance leg moves forwards and backwards to compensate the gravity motion.

During the third interval, the supporting leg extends itself slightly further whereas the hip joint in the balance leg pushes the whole leg down. The negative torque shown by the knee joint does not indicate the bending of the knee. Instead, it shows the reaction of the knee control system to the pushing down of the hip joint to control the fall of the robot.

During the interval 0.13 to $0.15 \mathrm{~s}$, both feet are on the ground. The reaction forces applied at the time of the ground impact lead to the large torques shown for the supporting leg mainly because: (i) it occurs earlier than expected (due to the approximations made on the dynamics model) and (ii) at that time the leg was still pushing forward opposing to the ground reaction forces. To help the waist recovering the original height, the knee in the balance leg slightly bends from its landing position while the ankle compensates by slightly extending its foot.

Figure 8 shows the forces applied by the prismatic actuators in the RPRRPR structure. The torques values for the hip and ankle joints are similar to the corresponding ones in the $6 \mathrm{R}$ structure and hence their interpretation is herein ommited. As before, the experiment time horizon is divided into four intervals following an initialization period.

During the initialization period, the support and balance knees generate constant forces of similar value and opposite signs (the support knee pushing up and the balance knee pushing down to compensate for the ground reaction force). In interval 1 , the balance knee starts shortening its length to help raising the balance leg. The support knee exhibits a slight reaction to the motion of the balance knee. The small glitches aroung $0.065 \mathrm{~s}$ are caused by the balance foot leaving the ground. In interval 2, the balance knee decreases the speed of its upward motion and the support knee is still showing its reaction to the upward motion of the balance knee. In interval 3, the balance knee starts moving down whereas the support knee reacts to this motion from the balance knee. In interval 4, the balance leg starts decreasing the speed of its downward motion, preparing for the touchdown that occurs at, approximately, $0.13 \mathrm{~s}$, and causes the small glitches in the torque. The support knee still shows a similar pattern of reaction to the down motion of the balance knee.

Note that, during the balance phase, the force in the balance leg knee is much higher than the force necessary in the supporting phase. This is mainly due to the strong coupling between the revolution joints of the hip and ankle, but also to the required fast lifting and lowering of the foot.

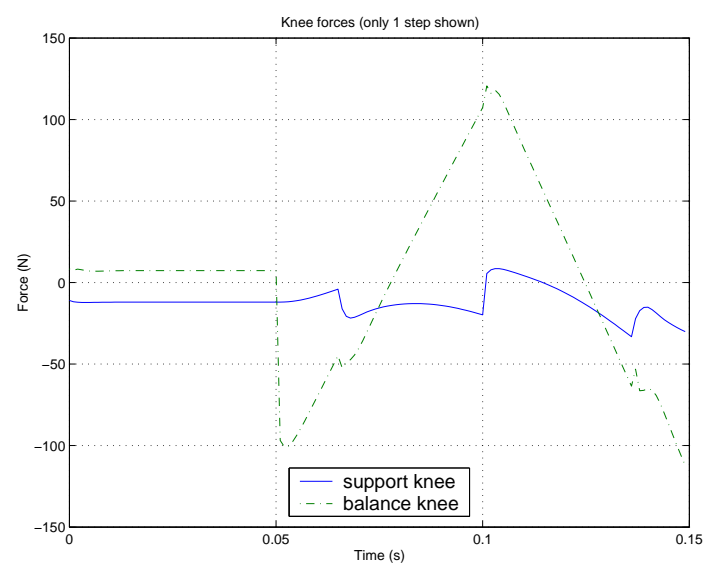

Fig. 8. Structure RPRRPR - Prismatic actuators forces 


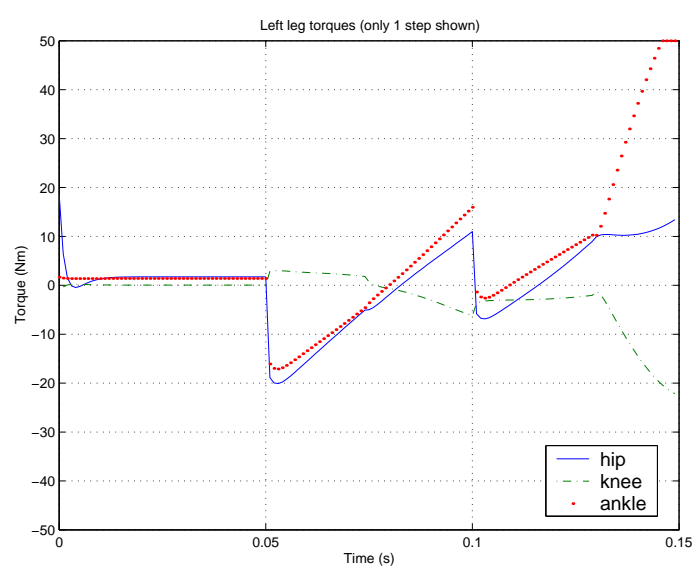

a) Supporting leg joint torques

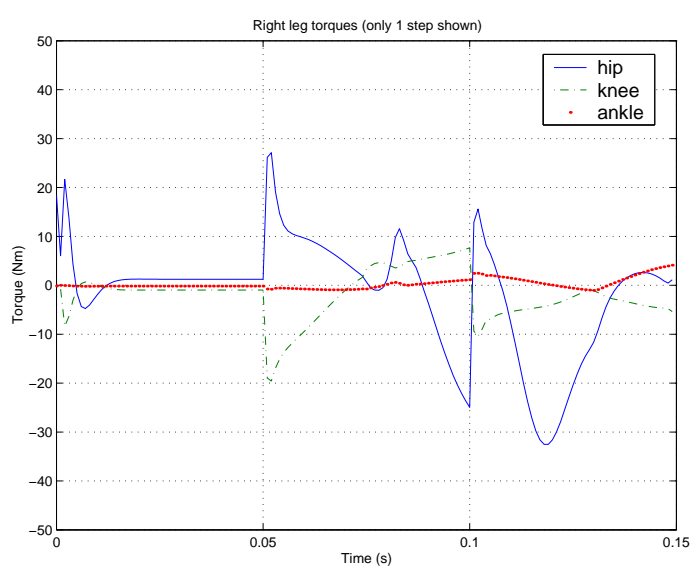

b) Balance leg joint torques

Fig. 7. Structure $6 \mathrm{R}$ - Joint torques

\section{CONCLUSIONS}

The two structures presented, though mechanically simple, cannot stand on only one foot, i.e., are statically unstable. For this reason, all the motions performed with only one foot on the ground must be fast, to prevent the robot from falling down. Because the robot moves at a relatively high speed, the actuators must be powerful enough to provide high torques/forces at high speeds. However, the values obtained from the simulation experiments can be matched by commercial off the shelf actuators, even in terms of mass. This means that both structures can be physically implemented.

Given the simplifying assumptions, the proposed dynamics modelling strategy is not exact. This caused the difference between the expected (i.e., defined by the reference trajectories) and the obtained step duration. However, the simulation results show that a standard partitioned robust control system was able to overcome most of the effects of the modelling errors (e.g., the reaction of the supporting leg to the motion of the balance leg to avoid excessive roll over) and provide a stable walking.

Since the motion specification for the RPRRPR structure is less restrictive, the movements of the hip and ankle joints of this structure have smaller amplitudes than those of the $6 \mathrm{R}$ structure. Therefore, for implementation purposes, the prismatic structure is preferred.

Further work includes (i) the assessment of alternative robust control strategies, e.g., using neural networks as in (D. Katić and M. Vukobratović, 2003), for the very simplified modeling scheme considered, (ii) studying the analytical stability for these structures without the point mass assumption, (iii) testing the inclusion of additional joints in the legs to improve the locomotion capabilitites, namely in what concerns different terrain types, (iv) adding a balance structure to improve the static stability of the structures, and (v) testing alternative reference trajectories used to generate the human like walking behavior aiming at obtaining smaller torques.

\section{REFERENCES}

Atsuo Takanishi, Hun-ok Lim, Masayuki Tsuda and Ichiro Kato (1990). Realization of dynamic biped walking stabilized by trunk motion on a sagittally uneven surface. In: Procs. of the IROS'90.

C. Chanchai and C. Prabhas (2001). Automatic synthesis of robot programs for a biped static walker by evolution computation. In: Procs. Second Asian Symposium on Industrial Automation and Robotics.

Carlos Canudas de Wit, Bruno Siciliano and Georges Bastin (1997). Theory of Robot Control. 2nd ed.. Springer.

Ching-Long Shi (1999). Ascending and descending stairs for a biped robot. IEEE Trans. on Systems, Man and Cybernetics, Part A: Systems and Humans.

D. Katić and M. Vukobratović (2003). Survey of intelligent control techniques for humanoid robots. Journal of Intelligent and Robotic Systems 37(2), 117141.

Eturoo Igarishi and Tooru Nogai (1992). Dynamic analysis and control of biped locomotion in the double supporting phase. Advanced Robotics.

Gill A. Pratt (2000). Legged robots at mit: What's new since raibert. IEEE Robotics and Automation Magazine.

J. Craig (1989). Introduction to Robotics. AddisonWesley.

J. Pratt and G. Pratt (1998). Intuitive control of a planar bipede walking robot. In: Procs. of the ICRA'98.

J. Pratt and G. Pratt (1999). Exploiting natural dynamics in the control of a $3 \mathrm{~d}$ bipedal walking simulation. In: Procs. of the CLAWAR99.

Yasutaka Fujimoto and Atsuo Kawamura (1998). Simulation of an autonomous biped walking robot including environmental force interaction. IEEE Robotics and Automation Magazine.

Youji Nakajima, Akira Yonemura and Atsuo Kawamura (2000). Experimental approach for the fast walking by the biped walking robot mari-1. AMC. 